\title{
Minimal Residual Disease and Survival Outcomes in Patients with Mantle Cell Lymphoma: a systematic review and meta-analysis
}

\author{
Yu Zhou, Haizhu Chen, Yunxia Tao, Qiaofeng Zhong, Yuankai Shi ${ }^{\circledR}$ \\ Department of Medical Oncology, National Cancer Center/National Clinical Research Center for Cancer/Cancer Hospital, Chinese Academy of Medical \\ Sciences \& Peking Union Medical College, Beijing Key Laboratory of Clinical Study on Anticancer Molecular Targeted Drugs, Beijing, 100021, China. \\ $\triangle$ Corresponding author: Yuankai Shi, Department of Medical Oncology, National Cancer Center/National Clinical Research Center for Cancer/Cancer \\ Hospital, Chinese Academy of Medical Sciences \& Peking Union Medical College, Beijing Key Laboratory of Clinical Study on Anticancer Molecular Targeted \\ Drugs, No. 17 Panjiayuan Nanli, Chaoyang District, Beijing, 100021, China. Tel.: +86-10-87788293; Fax: +86-10-87778781; E-mail: syuankai@cicams.ac.cn. \\ (c) The author(s). This is an open access article distributed under the terms of the Creative Commons Attribution License (https://creativecommons.org/licenses/by/4.0/). \\ See http://ivyspring.com/terms for full terms and conditions.
}

Received: 2020.08.14; Accepted: 2020.10.18; Published: 2021.01.01

\begin{abstract}
Background: Minimal residual disease (MRD) has shown the prognostic value in mantle cell lymphoma (MCL). To quantify the relationships between progression free survival (PFS) and overall survival (OS) with MRD status in MCL, we conducted this meta-analysis.

Methods: We searched databases including Pubmed, Embase, Web of Science and the Cochrane Library up to July 15th, 2020. Data of patients' characteristics, MRD assessment and survival outcomes were extracted and analyzed.

Results: Ten articles were included. For the impact of post-induction MRD status on survival outcomes, MRD positive status was associated with worse PFS (HR=I.44; $95 \% \mathrm{Cl} I .27-\mathrm{I} .62 ; \mathrm{P}<0.0000 \mathrm{I})$ and $\mathrm{OS}(\mathrm{HR}=\mathrm{I} .30$; $95 \% \mathrm{Cl}$ I.03-I.64; $P=0.03$ ) compared with MRD negative status. Regarding the impact of post-consolidation MRD status on survival outcomes, MRD positivity predicted shorter PFS (HR=I.84; $95 \% \mathrm{Cl} 1.49-2.26$; $P<0.0000 \mathrm{I})$ and $\mathrm{OS}(\mathrm{HR}=2.38 ; 95 \% \mathrm{Cl} \mathrm{I} .85-3.06 ; P<0.0000 \mathrm{I})$ than MRD negativity.

Conclusions: This study indicated that MRD positivity after induction and consolidation treatments was associated with worse PFS and OS for MCL. MRD-based treatment strategies should be further explored in clinical trials and real-world practice.
\end{abstract}

Key words: minimal residual disease, mantle cell lymphoma, clinical outcome, prognosis, meta-analysis

\section{Introduction}

Mantle cell lymphoma (MCL) is an uncommon subtype of non-Hodgkin lymphoma (NHL), comprising about $3 \%$ of NHL [1]. Most patients are diagnosed with advanced stage disease, considered as incurable by conventional chemotherapies [2]. In recent years, the application of high-dose chemotherapies, chemoimmunotherapies and nonchemotherapeutic agents such as frontline induction treatment, as well as autologous stem cell transplantation (ASCT) consolidation, and rituximab maintenance after ASCT has improved long term survival of MCL [3-8]. However, although these therapeutic strategies bring survival benefits, most patients will still relapse eventually. Also, the toxicities and economic burden from consolidation and maintenance treatment can still be the problems $[9,10]$. Therefore, early assessment of treatment efficacy and response-guided therapy are necessary in MCL management.

Minimal residual disease (MRD) refers to the persistent traceable tumor cells in patient's peripheral blood (PB) or bone marrow (BM) after treatment. Recent years, MRD detection has shown specificity and sensitivity for diagnosis and efficacy assessment in NHL [11]. MRD represents the depth of molecular remission and provides evidence for early response of treatment efficacy. Previous studies showed that MRD assessment could predict survival outcomes in 
MCL [12-16]. Also, in some previous clinical trials, surveillance of MRD can monitor response to prior therapy and may inform the need for further consolidation or maintenance therapy in MCL $[17,18]$. Due to the low incidence of MCL and the complexity of conventional detection techniques, previous single studies regarding MRD and survival outcomes were mostly from relatively small sample size. To further understand the impact of MRD on survival outcomes in newly diagnosed MCL, we conducted this systematic review and meta-analysis.

\section{Methods}

This systematic review and meta-analysis was performed in accordance with the Preferred Reporting Items for Systematic reviews and Meta-Analyses (PRISMA) guidelines[19] and has been registered on International Prospective Register of Systematic Reviews (PROSPERO) (register ID: CRD42020192171).

\section{Literature search}

Literature search was performed for articles from electronic databases including Pubmed, Embase, Web of Science and the Cochrane Library up to June 15th, 2020, using the MeSH terms "neoplasm, residual" and "Lymphoma, Mantle-Cell" and the free text words "Minimal Residual Disease", "Residual Tumor", "Residual Cancer", "MRD", "Mantle Cell Lymphoma" and "MCL". The complete search strategies were described in supplementary Table S1.

\section{Eligibility and study selection}

Full-text articles in English of randomized controlled trials or patient cohort studies were included. The main eligible criteria were as following: (1) studies in which patients were with newly diagnosed MCL and received no prior treatment; (2) studies in which the association between MRD and survival outcomes was reported; (3) studies from which the data of interest could be extracted. When the studies reported data from the same patient cohort or the patient cohort was overlapping, the study with most updated and complete data would be included. Outcomes of interest were progression-free survival (PFS) and overall survival (OS). Data were analyzed for PFS and OS grouped by MRD detection time. Two investigators (YZ and HZC) worked independently to assess the eligibility of studies. If there was any disagreement, studies would be re-assessed by the third investigator (YXT).

\section{Data Extraction and Quality Assessment}

Two investigators (YZ and HZC) worked independently to extract data from included studies.
The following information was extracted from the publications if available: region of the study, study sample size, MRD source, MRD detection method, MRD detection time, MRD cut-off value, median follow-up time, patients' median age, treatment for induction, treatment for consolidation and treatment for maintenance. When extracting hazard ratio (HR) and $95 \%$ confidence interval (CI) for aggregation and comparison, we used the following methods to get information needed: (1) when the publications provided $\mathrm{HR}$ and $95 \% \mathrm{CI}$, we extracted the direct data; (2) when the publications provided number at risk, observed number $(\mathrm{O})$ of events and the differences (O-Es), or Kaplan-Meier curves of PFS and OS, we regenerated survival results using methodology described by Tierney et al. [20]. Engauge Digitizer software (version 11.1) was applied to extract coordinates of points on the curves. Studies were excluded if HR and 95\% CI cannot be extracted using above methods.

Newcastle-Ottawa quality assessment scale was applied to assess the selection and comparability and outcomes of study cohorts [21]. The total scores ranged from zero to nine points, with a score of lower than five indicating poor quality, five to seven indicating medium quality, and higher than seven representing high quality. Two investigators (YZ and HZC) worked independently to assess quality of the studies.

\section{Statistical Analyses}

The outcomes of interest were PFS and OS. For pooled analysis of studies reporting MRD status and survival outcomes, estimated survival curves were generated using methodology described by Combescure et al. [22]. HR and 95\% CI were used to conduct a pooled $\mathrm{HR}$ and $95 \% \mathrm{CI}$ for survival outcomes. Random-effects meta-analysis using DerSimonian and Laird method was applied to conduct pooled treatment effects from included studies [23]. Cochrane $Q$ test and $I^{2}$ statistic were applied to estimate the heterogeneity among included studies. For $Q$ test, $P$ value $<0.05$ indicated significant heterogeneity; for $I^{2}$ statistics, $I^{2}$ value $>50 \%$ suggest substantial heterogeneity [24]. Funnel plots, Begg's test and Egger's tests were used to evaluate the publication bias $[25,26]$. Trim and fill method were applied for testing and adjusting publication bias [27]. Sensitivity analysis using random-effects model was performed to detect the potential source of heterogeneity. $\mathrm{R}$ software (version 3.6.2, https:// www.R-project.org/) was applied for all the data analysis. Two-side $P$ value $<0.05$ was considered as statistically significant. 


\section{Results}

\section{Literature search and study selection}

In total, 719 records were identified and ultimately ten studies were considered eligible for quantitative meta-analysis $[4,12-16,28-31]$. The procedure of study selection was illustrated in Figure 1. Of the included ten studies, ten studies reported MRD-related PFS and six studies reported MRDrelated OS. Of note, the study by Pott et al. (2010) [13] aggregated data from two independent cohorts (MCL Younger Study and MCL Elderly Study) and data from MCL Younger Study was updated in the trial by Hermine et al. (2016) [4]. Hence, we only extracted data of MCL Elderly Study from the report by Pott et al. (2010).

For the quality of trials, included studies were assessed as low to moderated risk of bias. The results of Newcastle-Ottawa Scale for quality assessment were shown in supplementary Table S2.

\section{Patients' characteristics}

Median age of patients ranged between 55 to 73 years old across the ten studies. The induction therapies were multiple, including chemotherapy and chemoimmunotherapy. Six studies included ASCTeligible patients $[4,12,14-16,29]$ and in two of the six studies, patients received post-consolidation maintenance, using rituximab or bortezomib [14, 16]. Patients did not receive ASCT in the other four studies $[13,28,30,31]$ and in two of the four studies, patients received post-induction maintenance using interferon- $\alpha$ or rituximab [13, 30] (Table 1).

\section{MRD assessment}

As for the timing of MRD detection, in nine studies, MRD was assessed after the completion of induction treatments [4, 13-16, 28-31]. Four studies reported MRD status and the related survival outcomes after ASCT or during maintenance [4, 12, 13, 15]. The four studies monitored post-consolidation MRD status in similar protocol. In the trial by Pott et al. (2006) [12], post-consolidation MRD was monitored from months 3, 6, 9, and 12 after ASCT. In the trial by Hermine et al. (2016) [4], MRD status was assessed at clinical staging during every 3 months follow-up. In the trial by Kolstad et al. (2017) [15], MRD evaluation was performed at 2-3 months, 6 months post-ASCT and then every 6 months until relapse or 5 years follow-up was completed. In the trial by Pott et al. (2010) [13], MRD status was monitored at 2- to 3-monthly intervals during maintenance treatment. Only one study reported the impact of mid-induction treatment MRD status on survival outcomes [30]. Due to this reason, we did not perform meta-analysis for mid-induction MRD status.

Table I. Characteristics of included studies

\begin{tabular}{|c|c|c|c|c|c|c|c|c|c|c|c|c|}
\hline Study & Region & $\begin{array}{l}\text { MRD+ } \\
\text { pts } \\
\text { number }\end{array}$ & $\begin{array}{l}\text { MRD-pts } \\
\text { number }\end{array}$ & $\begin{array}{l}\text { MRD } \\
\text { source }\end{array}$ & $\begin{array}{l}\text { MRD } \\
\text { detection } \\
\text { method }\end{array}$ & $\begin{array}{l}\text { MRD cut- } \\
\text { off value }\end{array}$ & $\begin{array}{l}\text { Median follow- } \\
\text { up (months) }\end{array}$ & $\begin{array}{l}\text { Median age } \\
\text { (years) }\end{array}$ & $\begin{array}{l}\text { MRD related } \\
\text { outcomes }\end{array}$ & Induction & ASCT & Maintenance \\
\hline Howard 2002 & $\begin{array}{l}\text { North } \\
\text { America }\end{array}$ & 16 & 9 & $\mathrm{~PB}, \mathrm{BM}$ & PCR & $10^{-5}$ & 25.0 & 55 & PFS & R-CHOP & No & None \\
\hline Pott 2006 & Europe & 13 & 14 & $\mathrm{~PB}, \mathrm{BM}$ & PCR & $10^{-4}$ & 48.0 & 56 & PFS, OS & $\begin{array}{l}\mathrm{COP}, \mathrm{CHOP}, \\
\mathrm{PmM}\end{array}$ & Yes & None \\
\hline Pott 2010† & Europe & $\begin{array}{l}69 \ddagger \\
20 \S\end{array}$ & $\begin{array}{l}87 \ddagger \\
31 \S\end{array}$ & PB, BM & PCR, FC & $10^{-4}$ & 17.0 & 61 & PFS & R-CHOP, R-FC & No & $\begin{array}{l}\text { Interferon-a } \\
\text { or rituximab }\end{array}$ \\
\hline Gimenez 2012 & Europe & 8 & 9 & $\mathrm{~PB}, \mathrm{BM}$ & PCR & $10^{-5}$ & NR & 55 & PFS, OS & (R) $\mathrm{VAD}+\mathrm{C}$ & Yes & None \\
\hline Liu 2012 & $\begin{array}{l}\text { North } \\
\text { America }\end{array}$ & 21 & 18 & $\mathrm{~PB}, \mathrm{BM}$ & PCR & $10^{-4}$ to $10^{-5}$ & NR & 56 & PFS, OS & RM-CHOP & Yes & Rituximab \\
\hline Hermine 2016 & Europe & $\begin{array}{l}65^{\mathrm{a}} \\
55^{\mathrm{b}}\end{array}$ & $\begin{array}{l}126^{\mathrm{a}} \\
173^{\mathrm{b}}\end{array}$ & $\mathrm{PB}, \mathrm{BM}$ & PCR & $10^{-4}$ & 73.2 & 55 & PFS & $\begin{array}{l}\text { Alternating } \\
\text { R-CHOP/ } \\
\text { R-DHAP or } \\
\text { R-CHOP }\end{array}$ & Yes & None \\
\hline Kolstad 2017 & Europe & $\begin{array}{l}54^{\mathrm{a}} \\
23^{\mathrm{b}}\end{array}$ & $\begin{array}{l}76^{\mathrm{a}} \\
160^{\mathrm{b}}\end{array}$ & $\mathrm{PB}, \mathrm{BM}$ & PCR & $10^{-4}$ to $10^{-6}$ & 102.0 & 57 & PFS, OS & $\begin{array}{l}\text { Alternating } \\
\text { Maxi-CHOP-R/ } \\
\text { R-Ara-C }\end{array}$ & Yes & None \\
\hline Klener 2018 & $\begin{array}{l}\text { South } \\
\text { America }\end{array}$ & 22 & 30 & $\mathrm{~PB}, \mathrm{BM}$ & PCR & $10^{-4}$ & 54.0 & 70 & PFS, OS & $\begin{array}{l}\text { Alternation } \\
\text { R-CHOP/ } \\
\text { R-Ara-C }\end{array}$ & No & Rituximab \\
\hline Gressin 2019 & Europe & 11 & 35 & $\mathrm{~PB}, \mathrm{BM}$ & PCR & $10^{-5}$ & 52.0 & 73 & PFS, OS & RiBVD & No & None \\
\hline Kaplan 2020 & $\begin{array}{l}\text { North } \\
\text { America }\end{array}$ & 25 & 17 & $\mathrm{BM}$ & PCR & $10^{-4}$ to $10^{-5}$ & 99.6 & 59 & PFS, OS & RM-CHOP & Yes & Bortezomib \\
\hline
\end{tabular}

† Only data of MCL Elderly study was included in analysis.

¥Patients number after induction treatment.

s Patients number after ASCT or during first 12 months of maintenance treatment.

Abbreviations: pts, patients; MRD+, minimum residual disease positive; MRD-, minimum residual disease negative; $\mathrm{PB}$, peripheral blood; $\mathrm{BM}$, bone marrow; $\mathrm{PCR}$, polymerase chain reaction; R-CHOP, rituximab, cyclophosphamide, doxorubicin, vincristine and prednisone; $\mathrm{COP}$, cyclophosphamide, vincristine and prednisone; $\mathrm{CHOP}$, cyclophosphamide, doxorubicin, vincristine and prednisone; PmM, prednimustine, mitoxantrone; ASCT, autologous stem cell transplantation; FC, flow cytometry; R-DHAP, rituximab with high-dose cytarabine and cisplatin; R-FC, rituximab, fludarabine and cyclophosphamide; NR, not reported; (R)VAD+C, (rituximab) vincristine, doxorubicin, dexamethasone + chlorambucil; RM-CHOP, rituximab, methotrexate, cyclophosphamide, doxorubicin, vincristine, and prednisone; Maxi-CHOP-R, cyclophosphamide, doxorubicine, prednisolone, rituximab; R-Ara-C, rituximab, cytarabine; RiBVD, rituximab, bortezomib, bendamustine and dexamethasone. 
Peripheral blood and bone marrow were obtained for MRD assessment. One study adopted both polymerase chain reaction (PCR) and flow cytometry (FC) as detection method [13] while the other nine studies adopted PCR as detection method. All the included studies explored the association between survival outcomes and MRD with a minimum sensitivity of $10^{-4}$ (Table 1 ).

\section{Meta-analysis of post-induction MRD status}

To evaluate the impact of post-induction MRD status on PFS, data were extracted from nine studies involving 607 patients (246 MRD-positive patients; 361 MRD-negative patients). For OS, data were extracted from six studies involving 326 patients (141 MRD-positive patients; 185 MRD-negative patients). Compared with MRD-negative patients, MRDpositive patients had shorter PFS (HR=1.44; 95\% CI 1.27-1.62; $P<0.0001$; Figure $2 \mathrm{~A})$ and $\mathrm{OS}(\mathrm{HR}=1.30$; 95\%CI 1.03-1.64; $P=0.03$; Figure 2B). The pooled 5-year PFS for MRD-positive patients and MRD-negative patients were $42.8 \%$ (95\% CI $31.8 \%-57.6 \%$ ) and $68.9 \%$ (95\% CI 61.4\%-77.3\%) (Figure 3A). The pooled 5-year OS for MRD-positive patients and MRD-negative patients were $63.6 \%$ (95\%CI 54.8\%-73.8\%) and $82.3 \%$ $(95 \%$ CI $76.1 \%-88.9 \%)$ (Figure 3B). In the tests of heterogeneity, there were no significant differences among the included studies for PFS $\left(X^{2}=6.29, \mathrm{df}=8\right.$; $\left.P=0.61 ; I^{2}=0 \%\right)$ and $O S\left(X^{2}=3.02, d f=5 ; P=0.70 ; I^{2}=0 \%\right)$.

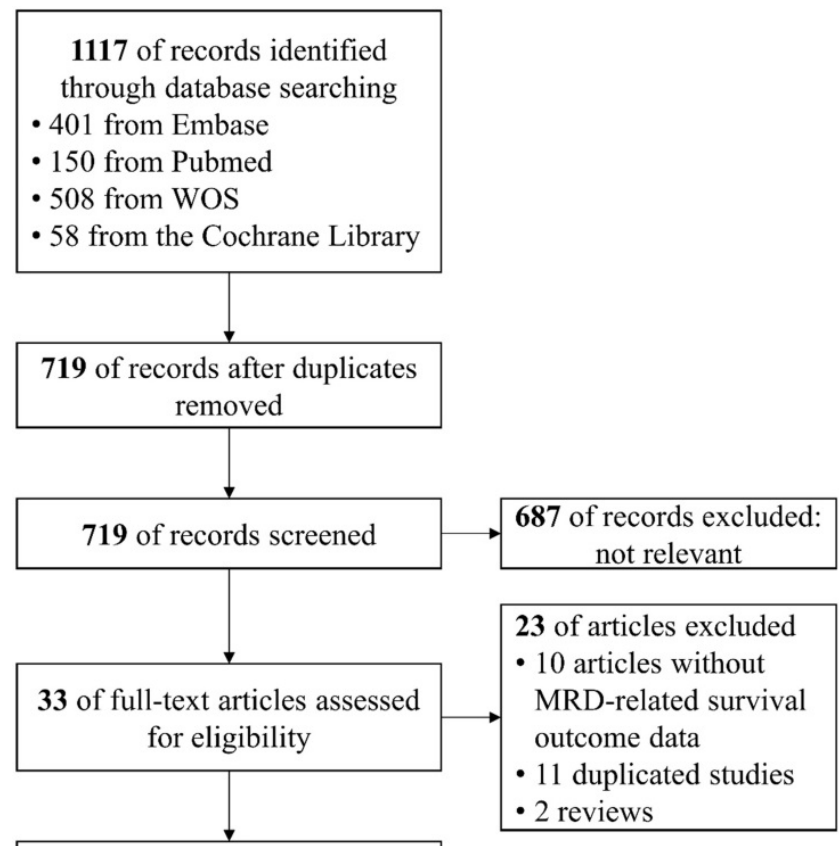

$\mathbf{1 0}$ of studies included in quantitative synthesis (metaanalysis)

Figure I. Flow chart of the search and screening process.
A

$\begin{array}{lrrr}\text { Study } & \text { HR } & 95 \% \mathbf{C l} & \text { Weight } \\ \text { Howard 2002 } & 0.96 & {[0.54 ; 1.73]} & 4.5 \% \\ \text { Pott 2010 } & 1.32 & {[0.61 ; 2.87]} & 2.5 \% \\ \text { Gimenez 2012 } & 1.40 & {[0.70 ; 2.78]} & 3.2 \% \\ \text { Liu 2012 } & 1.76[1.07 ; 2.90] & 6.2 \% \\ \text { Hermine 2016 } & 1.54 & {[1.29 ; 1.83]} & 48.6 \% \\ \text { Kolstad 2017 } & 1.28 & {[0.96 ; 1.72]} & 17.7 \% \\ \text { Klener 2018 } & 1.14 & {[0.78 ; 1.68]} & 10.3 \% \\ \text { Gressin 2019 } & 1.74 & {[0.75 ; 4.06]} & 2.1 \% \\ \text { Kaplan 2020 } & 1.93[1.11 ; 3.36] & 4.9 \%\end{array}$

Total $(95 \% \mathrm{Cl}) 1.44[1.27 ; 1.62] 100.0 \%$ Heterogeneity: $\mathrm{Tau}^{2}=0 ; \mathrm{Chi}^{2}=6.29, \mathrm{df}=8(\mathrm{P}=0.61) ; \mathrm{I}^{2}=0 \%$ Test for overall effect: $z=5.77, P<0.0001$

B

$\begin{array}{lrrr}\text { Study } & \text { HR } & \mathbf{9 5 \%} \text { Cl } & \text { Weight } \\ \text { Gimenez 2012 } & 1.03 & {[0.37 ; 2.93]} & 5.0 \% \\ \text { Liu 2012 } & 0.88 & {[0.35 ; 2.21]} & 6.4 \% \\ \text { Kolstad 2017 } & 1.26 & {[0.88 ; 1.79]} & 43.5 \% \\ \text { Klener 2018 } & 1.15 & {[0.70 ; 1.90]} & 21.7 \% \\ \text { Gressin 2019 } & 1.68 & {[0.82 ; 3.42]} & 10.7 \% \\ \text { Kaplan 2020 } & 1.93 & {[1.00 ; 3.72]} & 12.6 \%\end{array}$

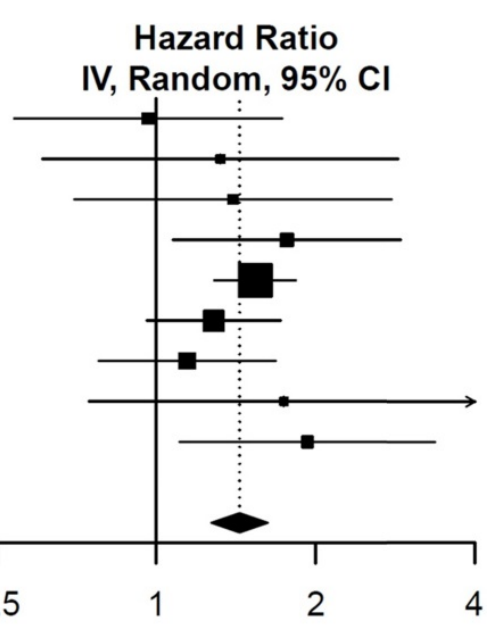

Hazard Ratio IV, Random, $95 \% \mathrm{CI}$

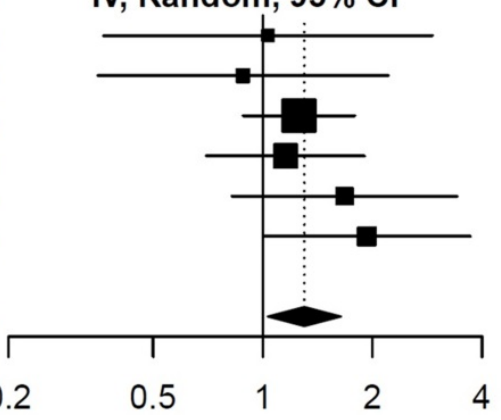

Figure 2. Forest plots for overall effect of post-induction minimal residual disease (MRD) status on (A) progression free survival (PFS) and (B) overall survival (OS). 

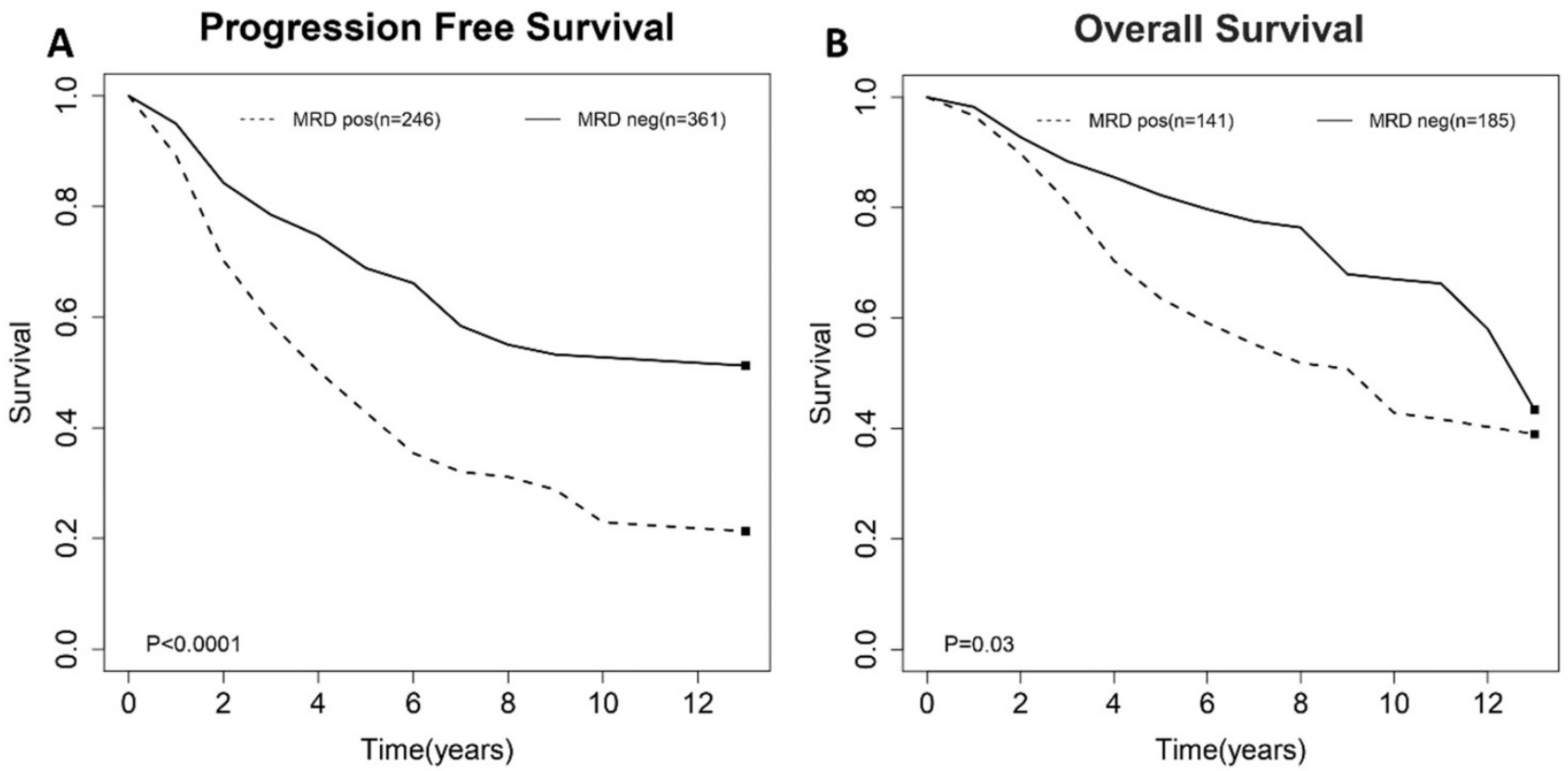

Figure 3. Estimated survival curves for (A) progression free survival (PFS) and (B) overall survival (OS) comparing post-induction minimal residual disease (MRD) positivity and negativity groups.

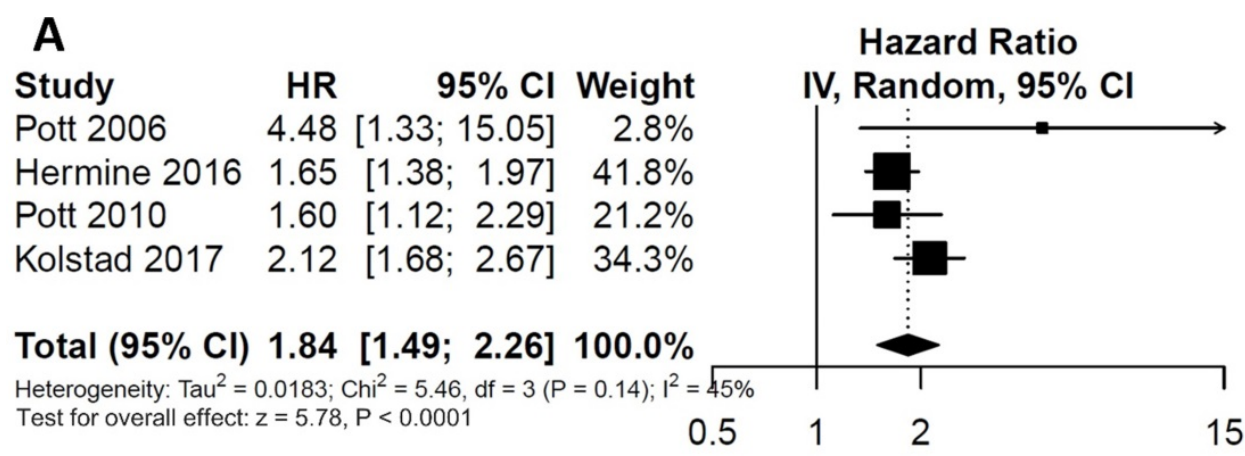

B

Study

Pott 2006

HR $\quad 95 \% \mathrm{Cl}$ Weight

Kolstad 2017

$2.93[1.19 ; 7.19] \quad 7.9 \%$

$2.34[1.80 ; 3.04] \quad 92.1 \%$

Total $(95 \% \mathrm{Cl}) 2.38[1.85 ; 3.06] 100.0 \%$

Heterogeneity: $\mathrm{Tau}^{2}=0 ; \mathrm{Chi}^{2}=0.22, \mathrm{df}=1(\mathrm{P}=0.64) ; \mathrm{I}^{2}=0 \%$

Test for overall effect: $z=6.74, P<0.0001$

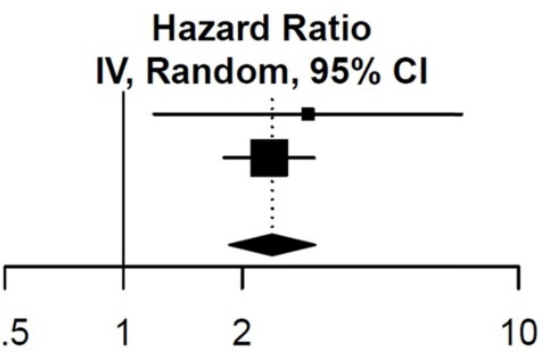

Figure 4. Forest plots for overall effect of post-consolidation minimal residual disease (MRD) status on (A) progression free survival (PFS) and (B) overall survival (OS).

\section{Meta-analysis of post-consolidation MRD status}

For the impact of post-consolidation MRD status on PFS, data from four studies involving 489 patients (111 MRD-positive patients; 378 MRD-negative patients) were extracted and analyzed. Concerning OS, data from 2 studies involving 210 patients (36 MRD-positive patients; 174 MRD-negative patients) were extracted. MRD positivity was associated with worse PFS (HR=1.84; 95\%CI 1.49-2.26; $P<0.0001$; Figure 4A) than MRD negativity, with a moderate heterogeneity among the four studies $\left(\chi^{2}=5.46, \mathrm{cdf}=3\right.$; $\left.P=0.14 ; \quad I^{2}=45 \%\right)$. The pooled 5-year PFS for MRD-positive patients and MRD-negative patients were $70.5 \%$ (95\% CI $65.5 \%-76.0 \%)$ and $28.4 \%$ (95\% CI 17.5-46.0\%) (Figure 5A). As there was a moderated heterogeneity among the studies for PFS, we performed a sensitivity analysis by removing one trial each time to explore the source of heterogeneity. By removing the trial by Kolstad et al., the heterogeneity of the studies for PFS reduced $\left(P=0.27 ; \mathrm{I}^{2}=24 \%\right)$. Patients with MRD positive status still tended to have shorter PFS (HR=1.67; 95\% CI 1.43-1.95; $P<0.0001)$. 


\section{A Progression Free Survival}

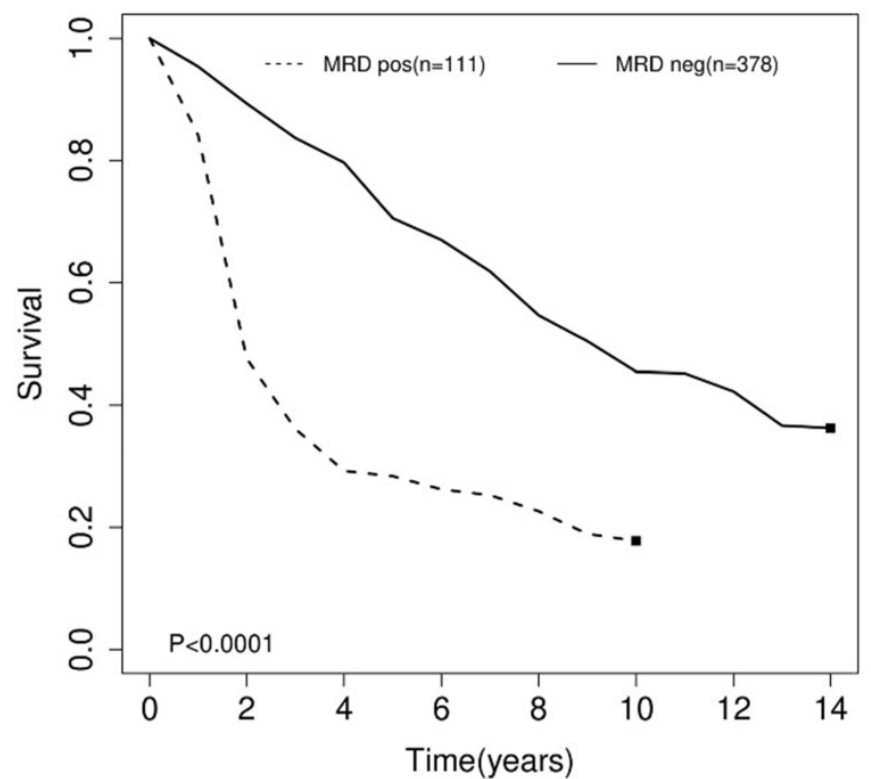

B Overall Survival

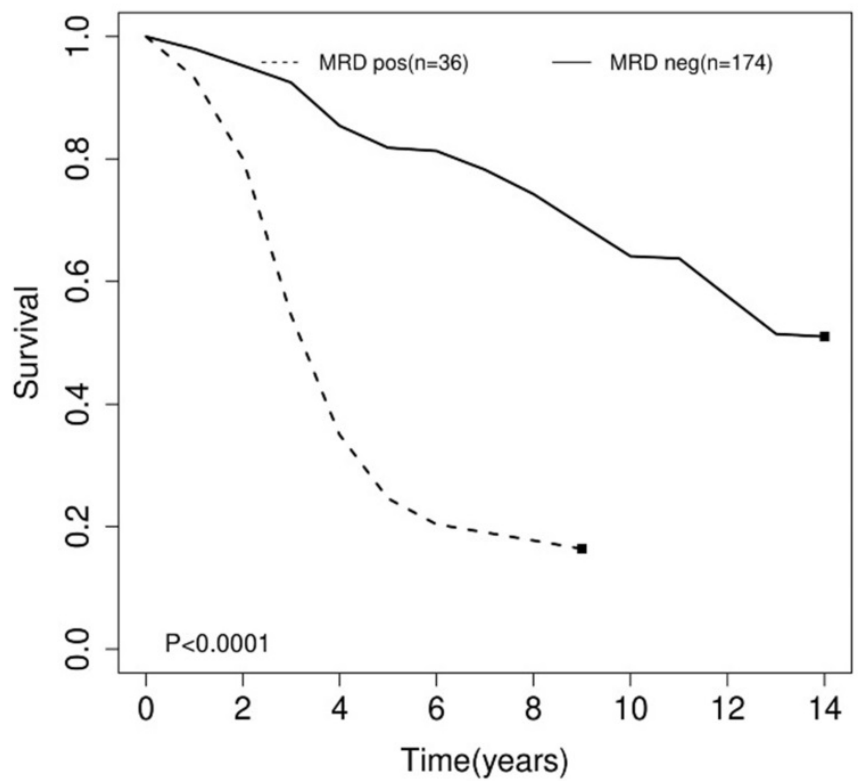

Figure 5. Estimated survival curves for (A) progression free survival (PFS) and (B) overall survival (OS) comparing post-consolidation minimal residual disease (MRD) positivity and negativity groups.

As for OS, compared with MRD negativity, MRD positivity was significantly associated with shorter OS $(\mathrm{HR}=2.38$; 95\%CI 1.85-3.06; $P<0.0001$; Figure 4B). There was no significant difference among studies for OS in the test of heterogeneity $\left(\chi^{2}=0.22, \mathrm{df}=1 ; P=0.64\right.$; $\left.I^{2}=0 \%\right)$. The pooled 5-year OS for MRD-positive patients and MRD-negative patients were $24.6 \%$ (95\%CI 11.9\%-51.0\%) and 81.1\% (95\%CI 75.5\%-88.6\%) (Figure 5B).

\section{Publication bias}

Funnel plots indicated no significant publication bias (Supplementary Figure S1A, 2A, 3A \& 4). In Begg's and Egger's tests, results showed no evidence of publication bias for the studies of post-induction MRD status for PFS (Begg's test: P=0.9195; Egger's test: $P=0.7388)$, post-induction MRD status for OS (Begg's test: $P=0.7500$; Egger's test: $P=0.2111$ ) and post-consolidation MRD for PFS (Begg's test: $P=0.7194$; Egger's test: $P=0.9701$ ) (supplementary Table S3). Considering the relatively small number of included studies in our meta-analysis $(n=10)$, small trial bias might exist. We performed trim and fill methods to adjust publication bias and regenerated funnel plots based on the adjusted results. The adjusted plots were showed in supplementary (Figure $\mathrm{S} 1 \mathrm{~B}, 2 \mathrm{~B}$ and $3 \mathrm{~B}$ ). Of note, due to the limited number of included studies reported post-consolidation MRD status on overall survival $(n=2)$, Begg's, Egger's test and trim and fill methods were not applicable for testing and adjusting publication bias of these two studies.

\section{Sensitivity analysis}

We performed sensitivity analysis by removing one study each time. The forest plots were showed that the pooled results were not significantly changed by any single study. Results were illustrated in supplementary Figure S5, 6, 7 and 8.

\section{Discussion}

In this meta-analysis, we performed quantitative synthesis to explore the association of MRD status on survival outcomes for newly diagnosed MCL. Results revealed that patients with MRD positive status had worse PFS and OS than patients with MRD negative status.

MRD status after both induction therapy and consolidation therapy showed prognostic value. This may provide information for deciding timing of MRD assessment. On the one hand, post-induction and post-consolidation MRD status assessment could be useful because it has prognostic value and may provide information for further therapy decisionmaking. There are some previous studies showed that rituximab consolidation post-ASCT could eliminate MRD and improve survival [17, 18, 32, 33].Therefore, surveillance of MRD status after ASCT can be helpful to discriminate patients with probable worse outcome and these patients can get survival benefits from further consolidation. On the other hand, as MRD implies the depth of molecular remission, continuous assessment of MRD status can be a useful tool for monitoring early relapse. At present, computed 
tomography (CT) and positron emission tomography (PET)/CT are the regular tools for assessing remission status, monitoring relapse and driving clinical treatment decisions. Our study results indicated that for MCL patients, MRD status at certain time had strong predictive power for relapse and death during the whole treatment procedure. Therefore, MRD assessment may be a supplement for the regular imaging response monitoring in the future. Although MRD status is of prognostic value based on this metaanalysis, the recommendation of clinical application of MRD, especially MRD-driven treatment decision, still needs to be validated in large prospective cohort study. In fact, there is an ongoing prospective randomized, phase III study focusing on the impact of treatment options on survival outcomes in MRD negative MCL patients (NCT03267433). In this study, MCL patients in MRD-negative first remission will be randomized to undergo autologous hematopoietic stem cell transplantation (auto-HCT) followed by rituximab maintenance group or rituximab maintenance alone (without auto-HCT). Survival results of patients who are MRD-negative and patients who are MRD-positive or MRDindeterminate prior to auto-HCT will be explored. This study will provide more information on the prognostic value of MRD status in MCL patients and the clinical efficacy of consolidation and maintenance treatment for patients with different post-induction MRD status.

The induction treatments varied across the included studies. For the analysis of post-induction MRD status, the consolidation and maintenance protocols also varied. Patients in the five included studies received ASCT while patient in the other four studies did not receive ASCT. Also, patients of four studies received maintenance treatment after induction or consolidation. Despite of the differences, all the studies showed a consistent effect of MRD on PFS and OS, confirmed by non-significant heterogeneity in Cochrane $Q$ test and $I^{2}$ statistic tests. This indicated that overall effect of MRD status did not be influenced by treatment. MRD can be a prognostic marker independent of the induction, consolidation, and maintenance treatments. However, the probability that negative results tend less likely to be reported always exists and this may cause inevitable bias in meta-analysis. We suggest that future clinical trials and real-world practice are necessary to further assess this question.

Only one of included study reported MRD status and survival outcomes in patients achieved conventional complete remission (CR). Due to limited data, we did not pool the results to assess the prognostic value of MRD status in clinical CR patients' subgroup. But some previous studies showed that MRD negativity was emerging as a significant therapeutic goal instead of clinical CR. A study based on the randomized intergroup trials of the EU-MCL Network showed that among 406 patients in remission 6 months after ASCT or end of induction, a positive MRD status in PB was highly associated with a shorter PFS and OS [34]. Seventy-six MRD-positive patients confirmed clinical relapse, with only 10 patients being MRD-negative. Another retrospective study included MCL patients who underwent ASCT in clinical CR between 1996 and 2011 [35]. Of 75 patients achieving CR, 11\% (8/75) were MRD-positive. MRD-positive was associated with worse PFS (adjusted HR 4.043; 95\%CI 1.429-11.442; $P=0.0085$ ) and OS (adjusted HR 3.68; $95 \% \mathrm{CI} 1.55-8.79 ; P=0.0033)$. These results indicate that instead of conventional CR, MRD can be an important endpoint in clinical trials.

We identified two conference abstracts that could not be included into this meta-analysis due to insufficient data and lack of peer-review. One abstract by Torka et al. explored prognostic value of MRD in a single-arm, open-label, multi-center phase II study [36]. 37 patients received O-HyperCVAD/MA (ofatumumab, cyclophosphamide, vincristine, doxorubicin, and dexamethasone alternating with highdose methotrexate and cytarabine) as induction treatment in this study. ASCT and post-ASCT rituximab maintenance were performed. MRD status was assessed in 28 of 37 patients. Results showed that MRD negativity after 2 cycles of induction treatment was associated with improved PFS $(P=0.04)$ and OS $(P=0.03)$. Another abstract by Smith et al. reported the results of ECOG1411 randomized phase II trial [37]. Patients were randomly assigned to receive BR (bendamustine and rituximab) with or without bortezomib as induction treatment, followed by rituximab with or without lenalidomide as consolidation treatment. MRD status after 3 cycles of induction treatment was assessed in 189 patients. Results showed that mid-induction MRD status was correlated with PFS $(P<0.01)$. One of the included studies in our meta-analysis also reported the association of mid-induction MRD status and survival outcomes [30]. However, in this study, MRD status had no significant relevance with PFS and OS. These results showed that early MRD remission might predict survival. But the utility of mid-induction MRD status still needs to be confirmed in future trials.

In this study, we did not analyze the survival results of different detection methods as all the included studies adopted PCR as detection method (Table 1). Currently, multiparameter flow cytometry (MFC) and real-time quantitative polymerase chain 
reaction (RQ-PCR) are both the methods of choice for MRD detection. MFC is based on immunophenotype and can be well performed in the diagnosis of lymphoma at short detection time and relatively low cost. However, compared with PCR, the lower sensitivity of MFC limited its use in the follow-up period [38, 39]. Based on IGH rearrangement or $\mathrm{t}(11 ; 14)$, RQ-PCR is a sensitive tool for detecting MRD in follow-up period and its sensitivity can reach up to $10^{-5}[12,40]$. But for patients without canonical translocations, the allele-specific oligonucleotide (ASO) primer design is necessary and this time-consuming procedure limited the applicability. Recently, the advent of next generation sequencing (NGS) technique provides another powerful tool for MRD detection. This is a well-developed technique and has been applied in recent clinical trials of MCL [37]. Results showed that NGS could be more sensitive than FC when detection material was available. Based on the results mentioned above, we suggest that when applying MRD assessment in clinical practice, the methods for MRD detection should be determined to specific circumstances and should follow standardized protocols.

There are limitations of our meta-analysis that should be considered. Analysis according to other variables, such as MRD cut-off value and type of induction therapies, were not performed due to the small number of studies in each subgroup. Also, as we could not get access to individual-level data, the pooled survival rates at certain time points were estimated and we could not draw strong conclusion of the survival probabilities.

\section{Conclusion}

In summary, results of this meta-analysis showed that MRD positivity after induction and consolidation treatments was associated with worse PFS and OS for MCL. MRD-based treatment strategies should be further explored in clinical trials and real-world practice.

\section{Abbreviations}

MCL: mantle cell lymphoma; NHL: nonHodgkin lymphoma; ASCT: autologous stem cell transplantation; MRD: minimal residual disease; $\mathrm{PB}$ : peripheral blood; BM: bone marrow; PFS: progression free survival; OS: overall survival; HR: hazard ratio; CI: confidence interval; PCR: polymerase chain reaction; FC: flow cytometry; CR: complete remission.

\section{Supplementary Material}

Supplementary figures and tables. http://www.jcancer.org/v12p0553s1.pdf

\section{Acknowledgements}

This work is supported by Chinese Academy of Medical Sciences (CAMS) Innovation Fund for Medical Sciences (CIFMS) (Grant no. 2016-I2M-1-001) and China National Major Project for New Drug Innovation (Grant no. 2017ZX09304015).

\section{Authors' contributions}

YKS conceived and designed the study; $Y Z, Q F Z$ and YXT searched literature. YZ and HZC extracted data and finished quality assessment. YZ, HZC and YXT analyzed and interpreted the data. $\mathrm{YZ}$ wrote the manuscript. YKS revised the manuscript. All authors have read and approved the final version of the manuscript.

\section{Competing Interests}

The authors have declared that no competing interest exists.

\section{References}

1. Teras LR, DeSantis CE, Cerhan JR, et al. 2016 US lymphoid malignancy statistics by World Health Organization subtypes. CA Cancer J Clin. 2016;66:443-59.

2. Teodorovic I, Pittaluga S, Kluin-Nelemans JC, et al. Efficacy of four different regimens in 64 mantle-cell lymphoma cases: clinicopathologic comparison with 498 other non-Hodgkin's lymphoma subtypes. European Organization for the Research and Treatment of Cancer Lymphoma Cooperative Group. J Clin Oncol. 1995;13:2819-26.

3. Epperla N, Hamadani M, Fenske TS, et al. Incidence and survival trends in mantle cell lymphoma. Br J Haematol. 2018;181:703-6.

4. Hermine O, Hoster E, Walewski J, et al. Addition of high-dose cytarabine to immunochemotherapy before autologous stem-cell transplantation in patients aged 65 years or younger with mantle cell lymphoma (MCL Younger): a randomised, open-label, phase 3 trial of the European Mantle Cell Lymphoma Network. Lancet. 2016;388:565-75.

5. Delarue R, Haioun C, Ribrag V, et al. CHOP and DHAP plus rituximab followed by autologous stem cell transplantation in mantle cell lymphoma: a phase 2 study from the Groupe d'Etude des Lymphomes de l'Adulte. Blood. 2013;121:48-53.

6. Le Gouill S, Thieblemont C, Oberic L, et al. Rituximab after Autologous Stem-Cell Transplantation in Mantle-Cell Lymphoma. N Engl J Med. 2017;377:1250-60.

7. Dreyling M, Lenz G, Hoster E, et al. Early consolidation by myeloablative radiochemotherapy followed by autologous stem cell transplantation in first remission significantly prolongs progression-free survival in mantle-cell lymphoma: results of a prospective randomized trial of the European MCL Network. Blood. 2005;105:2677-84.

8. Wang ML, Lee $\mathrm{H}$, Thirumurthi $\mathrm{S}$, et al. Ibrutinib-rituximab followed by Reduced chemo-immunotherapy consolidation in young, newly diagnosed mantle cell lymphoma patients: a window of opportunity to reduce chemo. Hematol Oncol. 2017;35:142-3.

9. Goyal RK, Nagar SP, Kabadi SM, et al. Adverse events, resource use, and economic burden associated with mantle cell lymphoma: a real-world assessment of privately insured patients in the United States. Leuk Lymphoma. 2019;60:955-63.

10. Kabadi SM, Near A, Wada K, et al. Treatment patterns, adverse events, healthcare resource use and costs among commercially insured patients with mantle cell lymphoma in the United States. Cancer Med. 2019;8:7174-85.

11. Chase ML, Armand P. Minimal residual disease in non-Hodgkin lymphoma current applications and future directions. Br J Haematol. 2018;180:177-88.

12. Pott C, Schrader C, Gesk S, et al. Quantitative assessment of molecular remission after high-dose therapy with autologous stem cell transplantation predicts long-term remission in mantle cell lymphoma. Blood. 2006;107:2271-8.

13. Pott C, Hoster E, Delfau-Larue $\mathrm{MH}$, et al. Molecular remission is an independent predictor of clinical outcome in patients with mantle cell lymphoma after combined immunochemotherapy: A European MCL intergroup study. Blood. 2010;115:3215-23.

14. Liu H, Johnson JL, Koval G, et al. Detection of minimal residual disease following Induction Immunochemotherapy predicts progression free survival in mantle cell lymphoma: Final results of CALGB 59909. Haematologica. 2012;97:579-85. 
15. Kolstad A, Pedersen LB, Eskelund CW, et al. Molecular Monitoring after Autologous Stem Cell Transplantation and Preemptive Rituximab Treatment of Molecular Relapse; Results from the Nordic Mantle Cell Lymphoma Studies (MCL2 and MCL3) with Median Follow-Up of 8.5 Years. Biol Blood Marrow Transplant. 2017;23:428-35.

16. Kaplan LD, Maurer MJ, Stock W, et al. Bortezomib consolidation or maintenance following immunochemotherapy and autologous stem cell transplantation for mantle cell lymphoma: CALGB/Alliance 50403. American Journal of Hematology. 2020;95:583-93.

17. Andersen NS, Pedersen LB, Laurell A, et al. Pre-Emptive Treatment With Rituximab of Molecular Relapse After Autologous Stem Cell Transplantation in Mantle Cell Lymphoma. J Clin Oncol. 2009;27:4365-70.

18. Kolstad A, Pedersen LB, Eskelund CW, et al. Molecular monitoring and tailored strategy with pre-emptive rituximab treatment for molecular relapse; results from the nordic mantle cell lymphoma studies (MCL2 and MCL3) with median follow-up of 8.5 years. Blood. 2016;128.

19. Moher D, Liberati A, Tetzlaff J, et al. Preferred reporting items for systematic reviews and meta-analyses: the PRISMA statement. BMJ. 2009;339:b2535.

20. Tierney JF, Stewart LA, Ghersi D, et al. Practical methods for incorporating summary time-to-event data into meta-analysis. Trials. 2007;8:16.

21. Stang A. Critical evaluation of the Newcastle-Ottawa scale for the assessment of the quality of nonrandomized studies in meta-analyses. Eur J Epidemiol. 2010;25:603-5.

22. Combescure C, Foucher Y, Jackson D. Meta-analysis of single-arm survival studies: a distribution-free approach for estimating summary survival curves with random effects. Stat Med. 2014;33:2521-37.

23. DerSimonian R, Laird N. Meta-analysis in clinical trials. Control Clin Trials. 1986:7:177-88

24. Huedo-Medina TB, Sánchez-Meca J, Marín-Martínez F, et al. Assessing heterogeneity in meta-analysis: Q statistic or I2 index? Psychol Methods. 2006;11:193-206.

25. Begg CB, Mazumdar M. Operating characteristics of a rank correlation test for publication bias. Biometrics. 1994;50:1088-101.

26. Egger M, Davey Smith G, Schneider M, et al. Bias in meta-analysis detected by a simple, graphical test. BMJ. 1997;315:629-34.

27. Duval S, Tweedie R. Trim and fill: A simple funnel-plot-based method of testing and adjusting for publication bias in meta-analysis. Biometrics. 2000;56:455-63.

28. Howard OM, Gribben JG, Neuberg DS, et al. Rituximab and CHOP induction therapy for newly diagnosed mantle-cell lymphoma: Molecular complete responses are not predictive of progression-free survival. J Clin Oncol. 2002;20:1288-94.

29. Gimenez E, Chauvet M, Rabin L, et al. Cloned IGH VDJ targets as tools for personalized minimal residual disease monitoring in mature lymphoid malignancies; a feasibility study in mantle cell lymphoma by the Groupe Ouest Est d'Etude des Leucémies et Autres Maladies du Sang. Br J Haematol. 2012;158:186-97.

30. Klener P, Fronkova E, Kalinova $\mathrm{M}$, et al Potential loss of prognostic significance of minimal residual disease assessment after R-CHOP-based induction in elderly patients with mantle cell lymphoma in the era of rituximab maintenance. Hematol Oncol. 2018;36:773-8.

31. Gressin R, Daguindau N, Tempescul A, et al. A phase 2 study of rituximab, bendamustine, bortezomib and dexamethasone for first-line treatment of older patients with mantle cell lymphoma. Haematologica. 2019;104:138-46.

32. Brugger W, Hirsch J, Grunebach F, et al. Rituximab consolidation after high-dose chemotherapy and autologous blood stem cell transplantation in follicular and mantle cell lymphoma: a prospective, multicenter phase II study. Ann Oncol. 2004;15:1691-8.

33. Ferrero S, Monitillo L, Mantoan B, et al. Rituximab-based pre-emptive treatment of molecular relapse in follicular and mantle cell lymphoma. Ann Hematol. 2013;92:1503-11.

34. Pott $\mathrm{C}$, Macintyre E, Delfau-Larue M-H, et al. MRD Eradication Should be the Therapeutic Goal in Mantle Cell Lymphoma and May Enable Tailored Treatment Approaches: Results of the Intergroup Trials of the European MCL Network. Blood. 2014;124.

35. Cowan AJ, Stevenson PA, Cassaday RD, et al. Pretransplantation Minimal Residual Disease Predicts Survival in Patients with Mantle Cell Lymphoma Undergoing Autologous Stem Cell Transplantation in Complete Remission. Biol Blood Marrow Transplant. 2016;22:380-5.

36. Torka P, Reddy N, Groman A, et al. Ofatumumab plus Hypercvad/MA induction leads to high rates of minimal residual disease (MRD) negativity in patients with newly diagnosed mantle cell lymphoma, results of a phase 2 study. Blood. 2019;134

37. Smith M, Jegede O, Parekh S, et al. Minimal residual disease (MRD) assessment in the ECOG1411 randomized phase 2 trial of front-line bendamustine-rituximab (BR)-based induction followed by rituximab (R) \pm lenalidomide (L) consolidation for mantle cell lymphoma (MCL). Blood. 2019;134.

38. Cheminant $\mathrm{M}$, Derrieux $\mathrm{C}$, Touzart $\mathrm{A}$, et al. Minimal residual disease monitoring by 8-color flow cytometry in mantle cell lymphoma: an EU-MCL and LYSA study. Haematologica. 2016;101:336-45.

39. Böttcher S, Ritgen M, Buske S, et al. Minimal residual disease detection in mantle cell lymphoma: methods and significance of four-color flow cytometry compared to consensus IGH-polymerase chain reaction at initial staging and for follow-up examinations. Haematologica. 2008;93:551-9.
40. Andersen NS, Donovan JW, Zuckerman A, et al. Real-time polymerase chain reaction estimation of bone marrow tumor burden using clonal immunoglobulin heavy chain gene and bcl-1/JH rearrangements in mantle cell lymphoma. Exp Hematol. 2002;30:703-10. 